\title{
The Coming of Age of Nutrigenetics and Nutrigenomics
}

\author{
Jing X. Kang \\ Massachusetts General Hospital and Harvard Medical School, Boston, Mass., USA
}

In recent years, human health has begun to face a new type of crisis. While the greatest risks of mortality in previous centuries were attributed to acute infectious disease, chronic metabolic disease is rapidly becoming a more significant concern, particularly in developed nations. According to the World Health Organization [1], 65\% of the world's population now live in countries where obesity kills more people than diseases related to malnourishment. Medical advancements may have enabled us to outwit many of the pathogens that harmed us in the past, but now our most serious concerns are the diseases that arise from how we live. To address the rising prevalence of chronic diseases such as diabetes, obesity, heart disease, and cancer, it is imperative that we continue to develop our knowledge of the biological mechanisms that underlie these diseases and seek safer and more effective prevention and treatment. Needless to say, contributions from the fields of nutrigenetics and nutrigenomics are more critical now than ever before.

Our understanding of genetics has progressed in leaps and bounds over the past decades, the apex of which was the completion of the Human Genome Project in 2004 [2]. Genetics play an integral role in every type of disease, whether the relationship is one of inheritance or the product of gene-environment interactions. This greater understanding of genetics has provided insight into the etiology of many diseases as well as the types of interventions that may be successful. We now understand that a variety of environmental stimuli trigger changes in gene expression, and that these changes underlie disease; for example, a decrease in global DNA methylation can upregulate genes involved in disease, such as cancer-promoting genes, thereby heightening the risk of developing cancer [3-5]. Clearly, nutrition and diet constitute a major source of environmental input, and there is reason to believe that increases in metabolic disease are associated with recent changes to the human diet and its impact on the genome. 
The Western human diet today is fundamentally different than it was throughout the majority of human evolution $[6,7]$. The diet we consume is marked by increases in energy content, saturated fat, trans fat, omega- 6 fatty acids, and cereal grains, and concurrent decreases in our intake of fruits, vegetables, proteins and omega-3 fatty acids [6-8]. Modern humans consume more carbohydrates than their ancestors and have an omega- 6 to omega- 3 fatty acid ratio that favors omega- 6 by as much as $20: 1$. Evolutionarily, this ratio would have been closer to 1:1, and the discrepancy has profound physiological consequences [8]. Given that omega- 3 fatty acids are potent inhibitors of inflammation and omega- 6 are largely inflammatory, the effect of changes to this ratio is that our bodies are subjected to a highly inflammatory environment [9]. Inflammation has been linked to a variety of diseases including cancer, cardiovascular and metabolic disorders, suggesting that this particular nutritional environment is at the crux of the global health crisis in chronic disease.

The fields of nutrigenetics and nutrigenomics are uniquely suited to addressing the detrimental gene-environment interactions that come forth as a result of our changing dietary landscape. For both fields, providing increasingly personalized nutritional recommendations is critical and should be a priority for future research. In terms of nutrigenetics, which describes the role of inheritance on the metabolism and processing of nutrients, we must account for how an individual's genetic background will shape the risks and benefits of consuming different types of foods and nutrients. Nutrigenomics, which describes how diet can impact gene expression and stability, should seek to provide recommendations on how to use diet to normalize gene expression for the purpose of reducing disease and aiding recovery from disease, as well as to promote continued good health for those who are disease free.

We will undoubtedly face certain challenges in achieving these goals. Personalized nutrition necessitates gathering detailed information about a population or an individual's genome, which, at present, is not always feasible. Moreover, obtaining genetic information always presents ethical considerations that we are still learning to assess and cope with in the medical community. The development of personalized nutrition recommendations relies on the various 'omic' technologies that have surfaced over the past few years [3, 4]. Metabolomics, lipidomics, proteomics, etc. enable us to assess health from the standpoint of the cellular and molecular events that are part of the disease process, and allow us to identify biomarkers that could potentially aid in the identification and treatment of disease. As we move forward, it is my hope that nutrigenetics and nutrigenomics will serve a pivotal role in biomedicine that ultimately promotes health, enables us to better treat and prevent disease, and extends life expectancy across the globe.

\section{References}

World Health Organization: Global Health Risks: Mortality and Burden of Disease Attributable to Selected Major Risks. Geneva, WHO Press, 2009.

2 International Human Genome Sequencing Consortium: Finishing the euchromatic sequence of the human genome. Nature 2004;431:931-945.

-3 Fenech M, El-Sohemy A, Cahill L, Ferguson LR, French TA, et al: Nutrigenetics and nutrigenomics: viewpoints on the current status and applications in nutrition research and practice. J Nutrigenet Nutrigenomics 2011;4:69-89.

4 Mutch DM, Wahli W, Williamson G: Nutrigenomics and nutrigenetics: the emerging faces of nutrition. FASEB J 2005; 19:1602-1616.

5 Sharma S, Kelly TK, Jones PA: Epigenetics in cancer. Carcinogenesis 2010;31:27-36.

6 Eaton SB, Konner M: Paleolithic nutrition. A consideration of its nature and current implications. N Engl J Med 1985; 312:283-289.

7 Leaf A, Weber PC: A new era for science in nutrition. Am J Clin Nutr 1987;45:1048-1053.

8 Simopoulos AP: Evolutionary aspects of diet: the omega-6/omega-3 ratio and the brain. Mol Neurobiol 2011;44:203215.

-9 Weylandt KH, Kang JX: Rethinking lipid mediators. Lancet 2005;366:618-620. 